\title{
Brouwer Fixed Point Theorem in the General Case
}

\author{
Karol Pąk \\ Institute of Informatics \\ University of Białystok \\ Poland
}

Summary. In this article we prove the Brouwer fixed point theorem for an arbitrary convex compact subset of $\mathcal{E}^{n}$ with a non empty interior. This article is based on [15].

MML identifier: BROUWER2, version: $\underline{7.11 .07 \quad 4.160 .1126}$

The notation and terminology used here have been introduced in the following papers: [17], [12], [1], [4], [7], [16], [6], [13], [10], [2], [3], [14], [9], [20], [18], [8], [19], [11], [21], and [5].

\section{Preliminaries}

For simplicity, we adopt the following convention: $n$ is a natural number, $p$, $q, u, w$ are points of $\mathcal{E}_{\mathrm{T}}^{n}, S$ is a subset of $\mathcal{E}_{\mathrm{T}}^{n}, A, B$ are convex subsets of $\mathcal{E}_{\mathrm{T}}^{n}$, and $r$ is a real number.

Next we state several propositions:

(1) $(1-r) \cdot p+r \cdot q=p+r \cdot(q-p)$.

(2) If $u, w \in$ halfline $(p, q)$ and $|u-p|=|w-p|$, then $u=w$.

(3) Let given $S$. Suppose $p \in S$ and $p \neq q$ and $S \cap$ halfline $(p, q)$ is Bounded. Then there exists $w$ such that

(i) $\quad w \in \operatorname{Fr} S \cap$ halfline $(p, q)$,

(ii) for every $u$ such that $u \in S \cap$ halfline $(p, q)$ holds $|p-u| \leq|p-w|$, and

(iii) for every $r$ such that $r>0$ there exists $u$ such that $u \in S \cap$ halfline $(p, q)$ and $|w-u|<r$. 
(4) For every $A$ such that $A$ is closed and $p \in \operatorname{Int} A$ and $p \neq q$ and $A \cap$ halfline $(p, q)$ is Bounded there exists $u$ such that Fr $A \cap$ halfline $(p, q)=\{u\}$.

(5) If $r>0$, then $\operatorname{Fr} \overline{\operatorname{Ball}}(p, r)=\operatorname{Sphere}(p, r)$.

Let $n$ be an element of $\mathbb{N}$, let $A$ be a Bounded subset of $\mathcal{E}_{\mathrm{T}}^{n}$, and let $p$ be a point of $\mathcal{E}_{\mathrm{T}}^{n}$. One can verify that $p+A$ is Bounded.

\section{Main Theorems}

Next we state four propositions:

(6) Let $n$ be an element of $\mathbb{N}$ and $A$ be a convex subset of $\mathcal{E}_{\mathrm{T}}^{n}$. Suppose $A$ is compact and non boundary. Then there exists a function $h$ from $\mathcal{E}_{\mathrm{T}}^{n}\left\lceil A\right.$ into $\operatorname{Tdisk}\left(0_{\mathcal{E}_{\mathrm{T}}^{n}}, 1\right)$ such that $h$ is homeomorphism and $h^{\circ} \operatorname{Fr} A=$ Sphere $\left(\left(0_{\mathcal{E}_{\mathrm{T}}^{n}}\right), 1\right)$.

(7) Let given $A, B$. Suppose $A$ is compact and non boundary and $B$ is compact and non boundary. Then there exists a function $h$ from $\mathcal{E}_{\mathrm{T}}^{n} \uparrow A$ into $\mathcal{E}_{\mathrm{T}}^{n}\left\lceil B\right.$ such that $h$ is homeomorphism and $h^{\circ} \operatorname{Fr} A=\operatorname{Fr} B$.

$(8)^{1}$ For every $A$ such that $A$ is compact and non boundary holds every continuous function from $\mathcal{E}_{\mathrm{T}}^{n}\left\lceil A\right.$ into $\mathcal{E}_{\mathrm{T}}^{n}\lceil A$ has a fixpoint.

(9) Let $A$ be a non empty convex subset of $\mathcal{E}_{\mathrm{T}}^{n}$. Suppose $A$ is compact and non boundary. Let $F_{1}$ be a non empty subspace of $\mathcal{E}_{\mathrm{T}}^{n} \uparrow A$. If $\Omega_{\left(F_{1}\right)}=\operatorname{Fr} A$, then $F_{1}$ is not a retract of $\mathcal{E}_{\mathrm{T}}^{n}\lceil A$.

\section{REFERENCES}

[1] Grzegorz Bancerek. Cardinal numbers. Formalized Mathematics, 1(2):377-382, 1990.

[2] Grzegorz Bancerek. The fundamental properties of natural numbers. Formalized Mathematics, 1(1):41-46, 1990.

[3] Grzegorz Bancerek. The ordinal numbers. Formalized Mathematics, 1(1):91-96, 1990

[4] Agata Darmochwał. Compact spaces. Formalized Mathematics, 1(2):383-386, 1990.

[5] Agata Darmochwał. Families of subsets, subspaces and mappings in topological spaces. Formalized Mathematics, 1(2):257-261, 1990.

[6] Agata Darmochwał. The Euclidean space. Formalized Mathematics, 2(4):599-603, 1991.

[7] Noboru Endou, Takashi Mitsuishi, and Yasunari Shidama. Convex sets and convex combinations. Formalized Mathematics, 11(1):53-58, 2003.

[8] Noboru Endou, Takashi Mitsuishi, and Yasunari Shidama. Dimension of real unitary space. Formalized Mathematics, 11(1):23-28, 2003.

[9] Krzysztof Hryniewiecki. Basic properties of real numbers. Formalized Mathematics, 1(1):35-40, 1990

[10] Stanisława Kanas, Adam Lecko, and Mariusz Startek. Metric spaces. Formalized Mathematics, 1(3):607-610, 1990.

[11] Artur Korniłowicz and Yasunari Shidama. Intersections of intervals and balls in $\mathcal{E}_{\mathrm{T}}^{n}$. Formalized Mathematics, 12(3):301-306, 2004.

[12] Artur Korniłowicz and Yasunari Shidama. Brouwer fixed point theorem for disks on the plane. Formalized Mathematics, 13(2):333-336, 2005.

[13] Yatsuka Nakamura, Andrzej Trybulec, and Czesław Byliński. Bounded domains and unbounded domains. Formalized Mathematics, 8(1):1-13, 1999.

\footnotetext{
${ }^{1}$ Brouwer Fixed Point Theorem
} 
[14] Beata Padlewska and Agata Darmochwał. Topological spaces and continuous functions. Formalized Mathematics, 1(1):223-230, 1990.

[15] Karol Sieklucki. Geometria i topologia. PWN, 1979.

[16] Andrzej Trybulec. Domains and their Cartesian products. Formalized Mathematics, 1(1):115-122, 1990.

[17] Andrzej Trybulec. A Borsuk theorem on homotopy types. Formalized Mathematics, 2(4):535-545, 1991.

[18] Wojciech A. Trybulec. Vectors in real linear space. Formalized Mathematics, 1(2):291-296, 1990.

[19] Zinaida Trybulec. Properties of subsets. Formalized Mathematics, 1(1):67-71, 1990.

[20] Edmund Woronowicz. Relations defined on sets. Formalized Mathematics, 1(1):181-186, 1990.

[21] Mirosław Wysocki and Agata Darmochwał. Subsets of topological spaces. Formalized Mathematics, 1(1):231-237, 1990.

Received December 21, 2010 